Case Report

\title{
Neuroendocrine tumour of colon mimicking psoas abscess: an unusual presentation of an uncommon malignancy
}

\author{
Sanjana Kumar*, Jainendra K. Arora
}

Department of General Surgery, Vardhman Mahavir Medical College and Safdarjung Hospital, New Delhi, India

Received: 08 February 2020

Accepted: 03 April 2020

\section{* Correspondence:}

Dr. Sanjana Kumar,

E-mail: sanjanabrook@gmail.com

Copyright: (c) the author(s), publisher and licensee Medip Academy. This is an open-access article distributed under the terms of the Creative Commons Attribution Non-Commercial License, which permits unrestricted non-commercial use, distribution, and reproduction in any medium, provided the original work is properly cited.

\begin{abstract}
Psoas abscess is a rare disease usually arising due to a benign underlying pathology such as tuberculous disease of spine, Crohn's disease and diverticulitis. But it can be an uncommon presentation of intra abdominal malignancy. Spontaneous rupture of colon cancer, combined with psoas abscess formation is very rare. Most of these cases have been reported in relation to carcinoma colon. Colon is an uncommon site for neuroendocrine tumors. $70-80 \%$ of these tumors are asymptomatic and are found incidentally at the time of surgery or on colonoscopy. Through this article we report a rare case of neuroendocrine tumor (NET) of colon in an elderly man presenting as psoas abscess associated with intestinal obstruction.
\end{abstract}

Keywords: Colon, Neuroendocrine tumor, Psoas abscess, Spontaneous rupture

\section{INTRODUCTION}

Psoas is a muscle of posterior abdominal wall. Due to its retroperitoneal location its involvement in pathology of intra-abdominal contents is extremely uncommon. ${ }^{1}$ Psoas abscess from colon cancer is usually preceded by perforation of cancer with fistula formation. ${ }^{2}$ Incidence of perforated colon cancer ranges from $3 \%$ to $10 \% .^{2}$

As NETs have an indolent course majority are asymptomatic at the time of discovery found incidentally at the time of surgery or other diagnostic evaluations. ${ }^{3,4}$ The classic carcinoid symptoms of cutaneous flushing and gut hypermotility occur in less than $10 \%$ of all patients, as the syndrome requires the presence of hepatic metastases. ${ }^{5}$ Symptoms that do occur tend to be vague and non-specific and are related to the mass effects of the tumor. ${ }^{5}$ These include vague abdominal pain, weight loss and constipation. ${ }^{5,6}$ There may be non-specific local effects of the tumor. ${ }^{7}$ For example, fibrosis from carcinoid tumors may cause obstruction from adhesions or stricturing of intestinal tumor and even mesenteric ischemia from constriction of mesenteric vessels. ${ }^{7}$

We performed a thorough search on various scientific research portal based on which there has never been a reported case of neuroendocrine tumor of colon presenting as psoas abscess.

\section{CASE REPORT}

A 45 year old gentleman presented to the surgical emergency with complaint of abdominal pain and distension since ten days with inability to pass flatus and stool since one day. Past history was significant for incision and drainage (I and D) done in right lower abdomen in view of right sided psoas abscess 20 days at an another hospital. There was no history of wheezing, flushing, weight loss, jaundice, hematochezia or melena.

On general physical examination, he was poorly nourished with presence of pallor and vital were stable. 
Per abdominal examination revealed generalized distension with I and D incision in right lumbar region draining seropurulent fluid. Emergency blood investigations were essentially normal except for mild anaemia (haemoglobin $9 \mathrm{~g} / \mathrm{dl}$ ). Chest X-ray was indicative of bilateral mild pleural effusion. Emergency ultrasonography of abdomen revealed circumferentially thickened ascending colon with thick echogenic mesentery and septated collection in peritoneal cavity due to perforation. Abdominal contrast-enhanced computed tomography (CECT) revealed a heterogeneous enhancement in the ascending colon suggesting malignancy with subheaptic and intraperitoneal collection with air foci within (Figure 1).

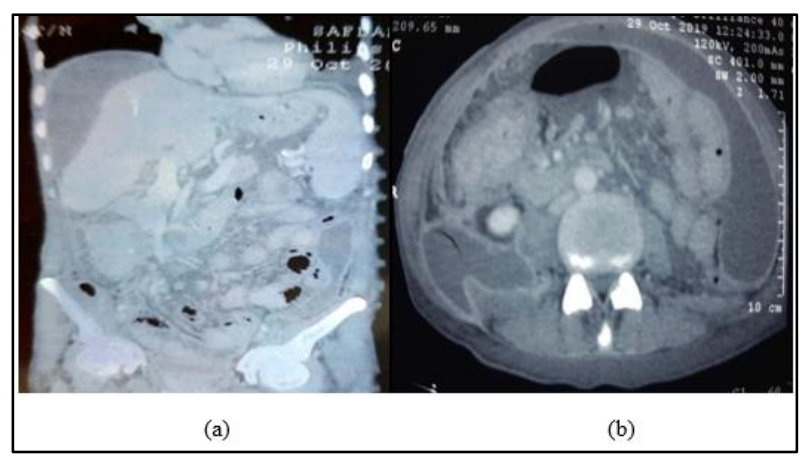

Figure 1: (a) CECT image (coronal view) heterogenously enhancing ascending colon lesion with subhehatic collection, (b) CECT image (axial view) showing ascending colon growth.

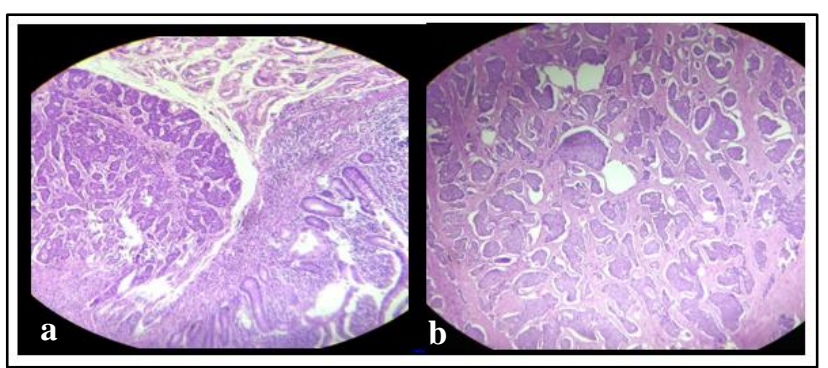

Figure 2: Hematoxylin and eosin-stained resected specimen showing nests and islands of tumor cells having eosinophic granular cytoplasm and rounded nuclei with stippled chromatin; (a) X100 magnification. (b) X200 magnification.

Emergency exploratory laparotomy was carried out which revealed an intraluminal growth in the ascending colon of size $12 \times 10 \mathrm{~cm}$ extending into caecum with no evidence of lymph node involvement. Right hemicolectomy was done, removing the tumor with a part of the colon. Histology confirmed a well differentiated neuroendocrine tumor, grade I (carcinoid) (Figure 2). Tumor was positive for neuron specific enolase (NSE), chromogranin, synaptophysin and CDX2 (Figure 3).

Postoperative period was uneventful. Patient was discharged on 10th post operative day and is planned for a follow-up.

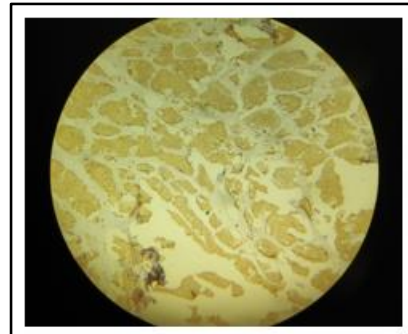

(A)

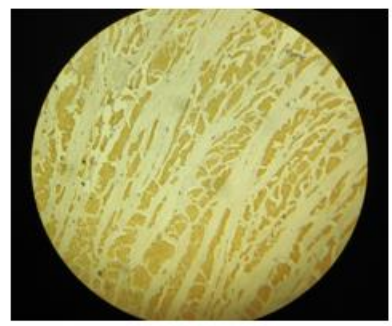

(C)

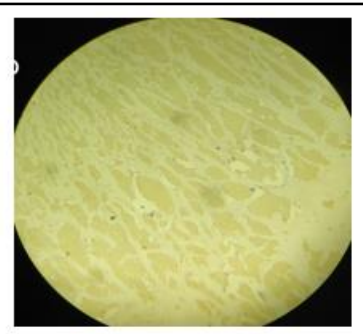

(B)

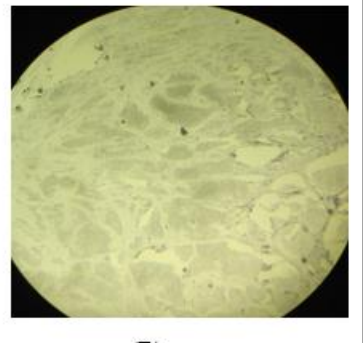

(D)
Figure 3: Colonic neuroendocrine carcinoma stained positive for (A) chromogranin (B) synaptophysin (C) CDX2 and (D) DOG - 1.

\section{DISCUSSION}

Neuroendocrine tumors of intestine arise from the kulchitsky cells or the enterochromaffin cells. ${ }^{6}$ They are also known as the amine precursor uptake and decarboxylation (APUD) cells. ${ }^{6}$ Patients with colonic carcinoid tumors most commonly present in the seventh decade of life with a slight female predominance. ${ }^{8}$ Upto $35-48 \%$ of colorectal carcinoids arise in the caecum and the ascending colon. ${ }^{9}$ These are classified under midgut carcinoids. ${ }^{9}$ Midgut carcinoid tumors are more likely to be symptomatic at the time of diagnosis, by which time the tumor has typically grown to greater than $5 \mathrm{~cm}$ in diameter. ${ }^{10}$ Late presentation of these tumor is due to the greater capacitance of the right colon compared with the distal colon and rectum. ${ }^{10}$ CECT scanning is considered the imaging modality of choice in these tumors to identify the site of disease and presence of lymphatic or visceral metastasis. ${ }^{6}$ Serum level of serotonin and other vasoactive peptides should be done, especially when there are symptoms suggesting carcinoid syndrome. ${ }^{8}$ Our patient did not have features of carcinoid syndrome.

Resection is the only curative treatment. Treatment is based on location and size of tumor and presence or absence of metastatic disease. For primary NETs smaller than $1 \mathrm{~cm}$ in diameter without evidence of regional lymph node metastasis, a segmental intestinal resection is adequate. For patients with lesions larger than $1 \mathrm{~cm}$, with multiple tumours or with regional lymph node metastasis require wide excision of involved bowel and mesentry. Lesion of terminal ileum, caecum and ascending colon are best treated by right hemicolectomy. ${ }^{6}$

Colonic carcinoids exhibit worse prognosis of all gastrointestinal carcinoid tumors. ${ }^{11}$ However, surgery 
provides complete cure when diagnosed early in the course of disease. ${ }^{6,11}$ These tumors have high propensity to recur and hence, long term follow up is necessary.

\section{CONCLUSION}

Psoas abscess is an extremely rare presentation in cases of spontaneous rupture of carcinoma colon. A strong sense of clinical suspicion is required in patients presenting with psoas abscess with continuous wound discharge and gastrointestinal complaints. Hence, it should always be in the minds of treating surgeon as a part of differential diagnosis in such patients.

Funding: No funding sources Conflict of interest: None declared

Ethical approval: Not required

\section{REFERENCES}

1. Cacurri A, Cannata G, Trastulli S. A rare case of perforated descending colon cancer complicated with a fistula and abscess of left iliopsoas and ipsilateral obturator muscle. Case Rep Surg. 2014;14:128506.

2. Yang JY, Lee JK, Cha SM, Joo YB. Psoas abscess caused by spontaneous rupture of colon cancer. Clin Orthop Surg. 2011;3(4):342-4.

3. Hay DJ, Curt JR. Carcinoid tumour as a complication of ulcerative colitis. Postgrad Med J. 1979;55:430-2.
4. Dodd SM. Chronic ulcerative colitis complicated by atypical carcinoid tumour. J Clin Pathol. 1986;39:913-6.

5. Ni SJ, Sheng WQ, Du X. Pathologic research update of colorectal neuroendocrine tumors. World $\mathrm{J}$ Gastroenterol. 2010;16(14):1713-9.

6. Courtney M, Townsend J. Sabiston Textbook of Surgery. The Biological Basis of Modern Surgical Practice, 20th edition. Elsevier. 2016.

7. Modlin IM, Mark K, Latich I, Zikusoka MN Shapiro MD. Current status of gastrointestinal carcinoids. Gasteroenterol. 2005;128(6):1717-51.

8. Obateru OA, Taiwo JA, Oladejo S, Awelimobor DA, Fadaunsi OO, Olokoba AB. Cecal carcinoid tumor in a Nigerian man: a case report and review of literature. Niger J Clin Pract. 2017;20:634-6.

9. Modlin IM, Lye KD, Kidd M. A 5-decade analysis of 13,715 carcinoid tumors. Cancer. 2003;97:93459.

10. Chung TP, Hunt SR. Carcinoid and neuroendocrine tumors of the colon and rectum. Clin Colon Rectal Surg. 2006;19(2):45-8.

11. Murray SE, Lloyd RV, Sippel RS, Chen H. Clinicopathologic characteristics of colonic carcinoid tumors. J Surg Res. 2013;184(1):183-8.

Cite this article as: Kumar S, Arora JK.

Neuroendocrine tumour of colon mimicking psoas abscess: an unusual presentation of an uncommon malignancy. Int Surg J 2020;7:1681-3. 